\title{
Un étau à endosser
}

\section{Thierry Aubry}

\section{(2) OpenEdition}

Journals

Édition électronique

URL : https://journals.openedition.org/rbnu/1662

DOI : $10.4000 /$ rbnu. 1662

ISSN : 2679-6104

\section{Éditeur}

Bibliothèque nationale et universitaire de Strasbourg

\section{Édition imprimée}

Date de publication : 1 novembre 2014

Pagination : 82-85

ISBN : 9782859230548

ISSN : 2109-2761

\section{Référence électronique}

Thierry Aubry, "Un étau à endosser », La Revue de la BNU [En ligne], 10 | 2014, mis en ligne le 01 novembre 2014, consulté le 18 mai 2021. URL : http://journals.openedition.org/rbnu/1662 ; DOI : https://doi.org/10.4000/rbnu.1662

\section{(C) $10(0$}

La Revue de la BNU est mise à disposition selon les termes de la Licence Creative Commons Attribution - Pas d'Utilisation Commerciale - Partage dans les Mêmes Conditions 4.0 International. 


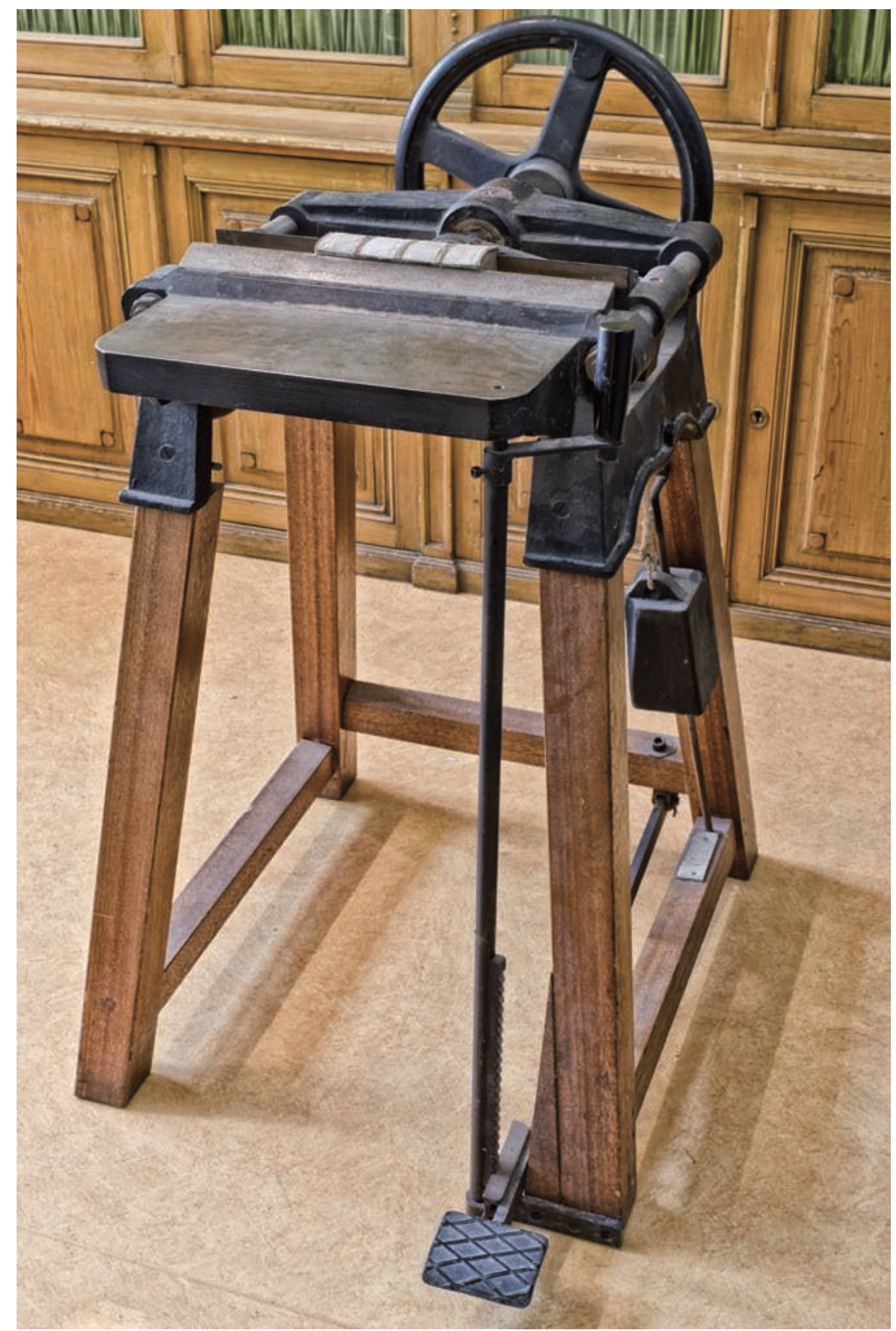




\section{UN ÉTAU À ENDOSSER}

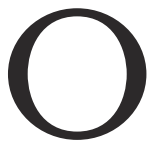

bjet atypique et incongru pour le commun des mortels, à l'instar de nombreux outils manufacturés au cours du $19^{e}$ siècle et de la révolution industrielle, l'étau à endosser partage le destin de ces outils anciens dont beaucoup sont devenus des énigmes, tout en constituant les fleurons de musées d'arts et de traditions populaires. L'étau à endosser n'a, pour sa part, pas encore achevé cette transition d'une fonction usuelle vers une destination muséographique et bien qu'étrange pour beaucoup, il fait encore partie du paysage des métiers du livre. Indispensable dans un atelier de reliure, on le trouve parfois aussi dans les ateliers de restauration, a fortiori dans les institutions qui ont spécialisé leurs activités dans ce dernier domaine. Il en est ainsi pour les ateliers de la Bibliothèque nationale de France, des Archives nationales ou de la Bibliothèque nationale et universitaire de Strasbourg. L'étau présenté ici fut probablement acquis lors de la création de l'atelier de reliure ${ }^{1}$, juste après la construction de la Kaiserliche Universitäts- und Landesbibliothek (ancêtre de la BNU) en 1871.

C'est un témoin, encore en activité, à la fois de la révolution industrielle et de l'âge d'or de l'artisanat de la reliure française. Bien que modifié par l'ajout d'une rotule mobile actionnant le contrepoids, l'étau de la BNU, sous cette forme d'acier et de fonte, muni d'un grand volant de serrage et d'une vis à filetage carré, avec ses volutes et son piètement en bois, reste familier à de nombreux relieurs car il est reproduit à l'identique par le dernier fabricant français de ces matériels ${ }^{2}$.

L'endossure - terme technique de la reliure, qui affectionne particulièrement cette terminaison (plaçure, couvrure, apprêture...) - correspond à l'étape située entre l'arrondissure du dos et la passure ${ }^{3}$ en carton. L'ouvrage est placé entre les deux mâchoires d'abord desserrées à l'aide du volant, pédale frontale relevée. Le relieur, le tenant d'une main, resserre l'étau à l'aide du volant jusqu'à ce qu'il soit maintenu. Ensuite, tenant la partie dépassant du dos à deux mains pour un réglage parfait, il serre fermement le livre en se servant de la pédale à pied.
Il s'agit ensuite, et c'est l'endossure, de créer le logement pour les cartons grâce à l'action répétée d'un marteau à pane légèrement arrondie qui, en partant du milieu de l'épaisseur du livre, rabattra les cahiers de part et d'autre sur chacune des deux mâchoires de l'étau afin de créer le mors, ou logement des cartons (ill. p. 85).

Auparavant, les cahiers ont été préalablement cousus les uns aux autres sur des supports de couture souvent appelés " nerfs " ou ficelles, avec un fil dont le diamètre est judicieusement choisi afin que le dos " monte " suffisamment pour qu'une fois placé dans l'étau, l'excédent d'épaisseur entre le dos et la gouttière (partie opposée au dos) soit rabattu de part et d'autre de celui-ci, sur chacune des mâchoires de l'outil, en formant un angle à $90^{\circ}$ (ill. p. 84, en bas, [d]). La taille de ce " logement " permettra d'y placer les cartons, ou plats du livre, sans que ceux-ci ne soient en surépaisseur. Il va sans dire que seule l'expérience professionnelle permet au relieur de choisir son fil en fonction du nombre de cahiers, de l'épaisseur et des dimensions (hauteur et largeur) de l'ouvrage, afin que l'épaisseur des mors et donc des cartons corresponde parfaitement, au dixième de millimètre près (ce qui demeure l'unité de mesure d'un bon relieur issu de la tradition du $19^{\mathrm{e}}$ siècle !).

Indiscutablement, l'étau (dont on attribue la conception, à la fin des années $1840^{4}$, à Jean Engel, le célèbre éditeur, et à Bernard Steimmetz, ingénieur et constructeur de machines à Paris) a été pensé pour améliorer techniquement et esthétiquement l'art de la reliure (selon les canons $d u 19^{\mathrm{e}}$ siècle), mais surtout pour accélérer son processus de fabrication à l'époque de l'apparition de la reliure d'éditeur, d'abord semi-industrielle avant de rapidement devenir exclusivement industrielle. Il reste donc un acteur de premier plan dans tous les ateliers de reliure " main " ${ }^{5}$, car inégalé quant à la précision et à la perfection du mors qu'il permet d'obtenir. Par contre, il a fait long feu dans le monde de la reliure industrielle en raison de l'action répétée et délicate, donc trop longue, du marteau. Il fut ainsi rapidement supplanté, au cours des années 1850, par d'autres modèles d'étaux ou presses 
à endosser. Citons celui de M. Pfeiffer, "mécanicien à Paris ", qui imagine un étau permettant d'endosser plusieurs livres en même temps ${ }^{6}$, ou encore, peu après, celui des américains Sauborn et Carter (ill. ci-contre, en haut), autorisant à la fois le travail d'arrondissure et d'endossure ${ }^{7}$ par le simple mouvement d'avant en arrière d'un cylindre sur le dos du livre, actionné par le bras situé au-dessus.

Toutefois, le mors plus approximatif obtenu par ce moyen reste un des indicateurs permettant de distinguer la reliure à la main, " traditionnelle ", de la reliure industrielle.

L'invention de cet outil, au milieu du $19^{\mathrm{e}}$ siècle, rompt avec un millénaire de pratique technique. Certes, les premiers ouvrages reliés en Occident, à savoir les reliures carolingiennes, n'étaient ni arrondis, ni endossés. Les ais, ou planches de bois (en chêne à cette époque) étaient parfois uniquement chanfreinés ${ }^{8}$ légèrement dans le mors. Pourtant, la technique d'arrondissure et d'endossure, d'abord légère sur les reliures romanes, puis plus prononcée sur les reliures gothiques, devient systématique à partir de la période moderne jusqu'à aujourd'hui (ill. cicontre, en bas).

Néanmoins, avant l'apparition de l'étau, cette étape avait toujours été d'une part peu précise, et d'autre part réalisée avec la seule aide d'un marteau. À ce titre, le degré de précision et la rapidité permis par l'invention de l'étau à endosser ont bel et bien été révolutionnaires.

Aujourd'hui, au sein des collections patrimoniales, la conservation préventive, la conservation curative et la restauration ont pris le pas sur la reliure, davantage externalisée ; l'étau de la BNU est donc moins utilisé qu'à l'époque de l'activité de l'atelier de reliure. Il reste toutefois un témoin de ce passé et contribue à la transmission de ce pan de l'histoire de la bibliothèque.

\section{Thierry Aubry}

\section{Notes}

1 - Cette datation nous a été confirmée par Gérard Péricaud, gérant de la société APG et ancien de la société Altenvet. Il est l'un des derniers, sinon le dernier artisan français fabricant d'étaux, de presses à percussion et de cisailles.

2 - En effet, G. Péricaud s'est servi d'un étau de la même série et du même fabricant pour reproduire et réaliser le modèle qu'il distribue aujourd'hui pour la société APG.

3 - Action de fixer les cartons sur le volume à relier, en passant les ficelles ou les rubans dans les trous pratiqués à cet effet.

4 - Voir R. Devauchelle, La reliure en France de ses origines à nos jours, tome II, 1960, p. 197

5 - La reliure " main " ou " faite à la main " se distingue de la reliure dite " industrielle ".

6 - Voir par exemple : Exposition de 1867. Délégation des ouvriers relieurs, zème partie : La reliure à l'exposition de 1867 . Etudes comparatives de la reliure ancienne et moderne, 1869 , p. 213 ; S. Lenormand., Nouveau manuel complet du relieur en tous genres (Manuel Roret), Paris, L. Mulo, 1830. M. Pfeiffer a présenté sa machine à endosser lors de l'exposition universelle internationale de 1855 (https://archive.org/stream/expositiondeoowynagoog/expositiondeoowynagoog_djvu.txt).

7 - Voir Exposition de $1867 . .$. (op. cit.)

8 - Un chanfrein consiste en un adoucissement de l'angle d'une planche à l'aide d'un rabot par exemple (cf. ill. du bas, figures [b] et [c]).

9 - J. A. Szirmai, The archeology of Medieval Bookbinding, Ashgate, Aldershot, 1999
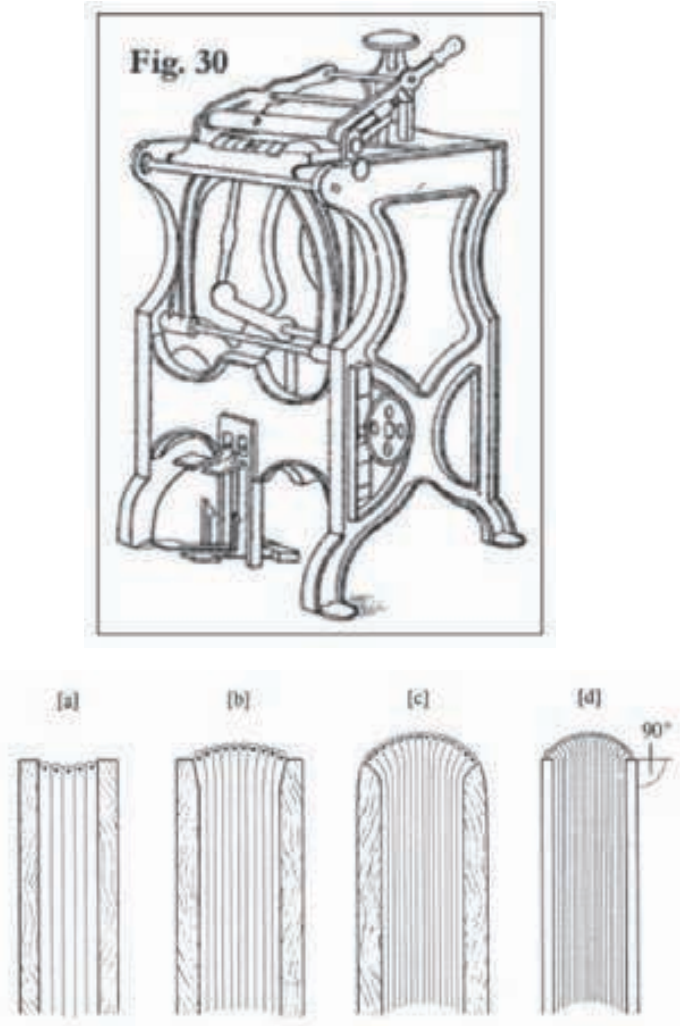

Évolution de l'arrondi des dos d'après J. A. Szirmai ${ }^{9}$ : [a] reliure carolingienne; [b] reliure romane ; [c] reliure gothique ; [d] reliure des périodes moderne et actuelle 


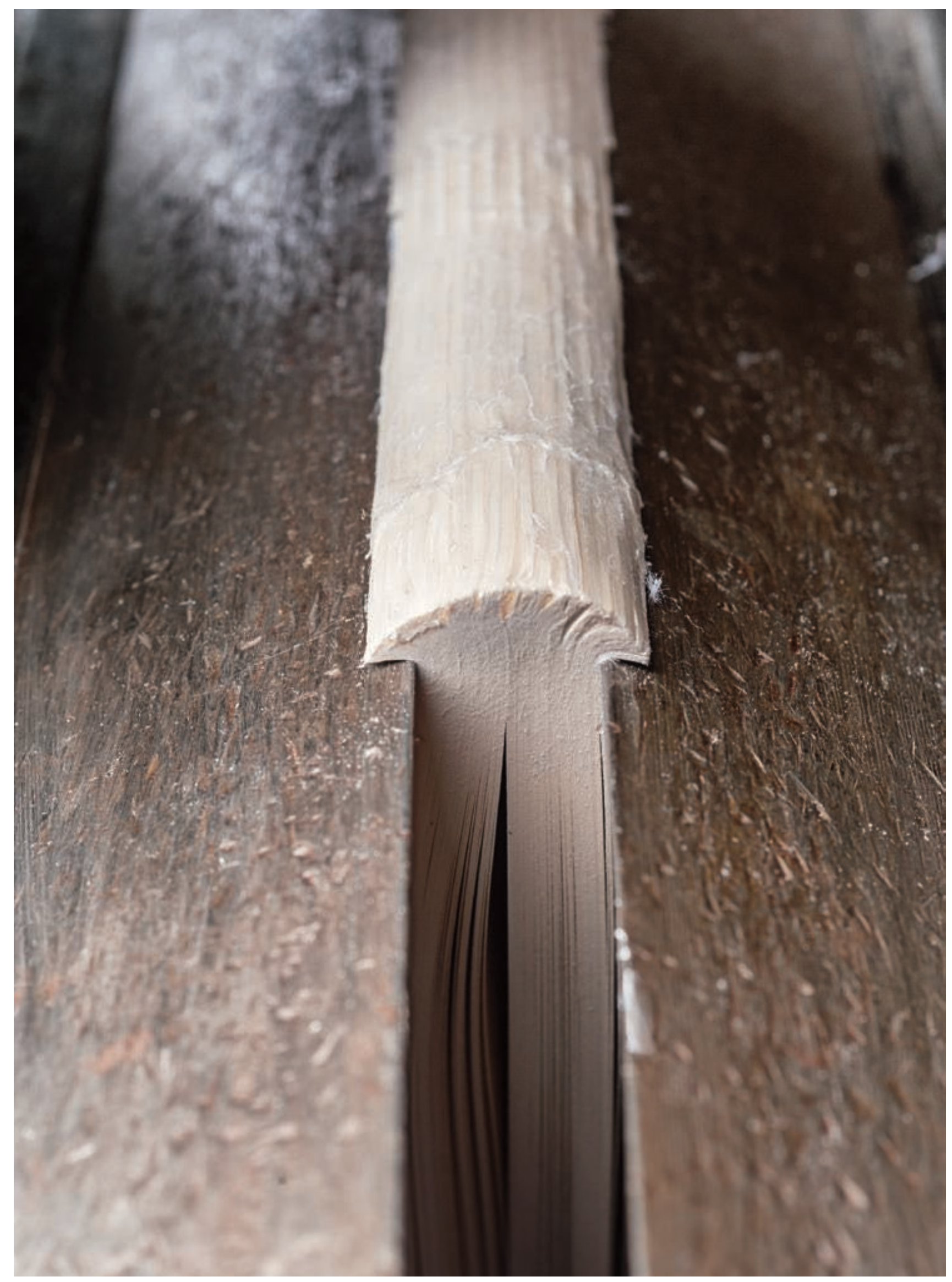

